\title{
Association of Treatment Intensity and Adherence to Lipid-Lowering Therapy with Major Adverse Cardiovascular Events Among Post-MI Patients in Germany
}

Ingo Ahrens · Artak Khachatryan · Bondo Monga · Eugen Dornstauder •

Eduard Sidelnikov

Received: January 7, 2021 / Accepted: March 3, 2021 / Published online: April 8, 2021

(C) The Author(s) 2021

\section{ABSTRACT}

Introduction: Patients with a history of myocardial infarction (MI) are at very high risk of subsequent cardiovascular events. This study evaluated the association of treatment intensity and adherence to lipid-lowering therapies (LLT) with major adverse cardiovascular events (MACE) among post-MI patients in Germany.

Supplementary Information The online version contains supplementary material available at https:// doi.org/10.1007/s12325-021-01697-8.

\section{Ahrens}

Department of Cardiology and Medical Intensive Care, Augustinerinnen Hospital, Academic

Teaching Hospital, University of Cologne, Cologne, Germany

A. Khachatryan

Certara, Evidence and Access (Previously-Analytica

Laser), London, UK

B. Monga

Elsevier, Berlin, Germany

B. Monga

Faculty of Medicine and School of Public Health,

University of Lubumbashi, Lubumbashi,

Democratic Republic of Congo

E. Dornstauder

Amgen $\mathrm{GmbH}$, Munich, Germany

E. Sidelnikov $(\square)$

Amgen (Europe) GmbH, Rotkreuz, Switzerland

e-mail: eduards@amgen.com
Methods: We carried out a retrospective cohort study using German health claims data (2010-2015). We included patients $\geq 18$ years, with a history of MI and who started an LLT (statin and/or ezetimibe), between 2011 and 2013. The follow-up period started 1 year after the second LLT prescription and continued until MACE, all-cause death or December 31, 2015, whichever occurred first. Treatment intensity was classified based on expected low-density lipoprotein cholesterol reduction; adherence was measured by the proportion of days covered using prescription data. A combined adherenceadjusted intensity variable was created by multiplying intensity and adherence. We used Cox proportional hazards models to control for age, sex, Charlson Comorbidity Index and other cardiovascular risk factors at baseline.

Results: A total of 14,944 patients were included. Mean age was $66.7(\mathrm{SD}=13.0)$ years; $68.7 \%$ of patients were men. Each $10 \%$ increase in treatment intensity, adherence, or adherence-adjusted intensity was associated with a decrease in the risk of MACE of $17 \%(\mathrm{HR}=0.83$, 95\% CI $0.79-0.87), 5 \% \quad(\mathrm{HR}=0.95,95 \%$ CI $0.94-0.97)$, and $14 \% \quad(\mathrm{HR}=0.86,95 \% \quad \mathrm{CI}$ 0.83-0.90), respectively.

Conclusions: Higher treatment intensity and/ or adherence of LLT was associated with significantly lower risk of MACE in post-MI patients. Strategies to tailor intensity to patient profiles and improve adherence could reduce the risk of cardiovascular events. 
Keywords: Cardiovascular disease; Lipidlowering therapy; Myocardial infarction; Statins; Treatment adherence; Treatment intensity

\section{Key Summary Points}

Patients with a history of myocardial infarction (MI) are at very high risk of subsequent cardiovascular events. Adequate lipid-lowering therapy (LLT) helps prevent such events.

In patients with a history of MI who started an LLT (statin and/or ezetimibe), each $10 \%$ increase in treatment intensity, adherence, or adherence-adjusted intensity is associated with a decrease in the risk of major adverse cardiovascular events (MI, stroke, or cardiovascular death) of $17 \%(\mathrm{HR}=0.83,95 \% \mathrm{CI}$ $0.79-0.87), 5 \%(\mathrm{HR}=0.95,95 \% \mathrm{CI}$ $0.94-0.97)$, and $14 \%(\mathrm{HR}=0.86,95 \% \mathrm{CI}$ 0.83-0.90), respectively.

Higher treatment intensity and/or adherence of LLT was associated with significantly lower risk of major adverse cardiovascular events in post-MI patients.

\section{DIGITAL FEATURES}

This article is published with digital features, including a summary slide, to facilitate understanding of the article. To view digital features for this article go to https://doi.org/10.6084/ m9.figshare.14061737.

\section{INTRODUCTION}

Cardiovascular disease (CVD) is a major driver of morbidity and mortality worldwide $[1,2]$, and is the most common cause of death in Germany [3]. Risk factors for CVD are multifactorial and include both modifiable (e.g. smoking, dyslipidaemia and lack of physical activity) and non-modifiable (e.g. type 2 diabetes or family history of cardiovascular disease) factors $[4,5]$. Elevated low-density lipoprotein cholesterol (LDL-C) is one of the most common, causal, major modifiable risk factors for the development of CVD [1]. Reducing LDL-C is part of a broader framework of CVD prevention in Europe, with lipid-lowering therapies (LLT) recommended as preventative measures against CVD $[1,6,7]$.

The effectiveness of LLT relies on both treatment intensity and adherence. Patients at risk for cardiovascular events may not receive the full benefit of LLT if intensity, adherence or both are suboptimal [8]. Current European and German guidelines focus on patients' CVD history, co-morbidities and LDL-C levels to guide the intensity of LLT. Patients with history of myocardial infarction (MI) are considered by the guidelines to be at very high risk for subsequent cardiovascular events. For these very high-risk patients, the guidelines recommend aggressive lowering of LDL-C by at least 50\% from the baseline level and below $1.4 \mathrm{mmol} / \mathrm{L}$ $[1,6,7]$. While higher intensity and adherence of LLT has been associated with greater reductions in LDL-C [9] and improved cardiovascular outcomes [10, 11], physicians may still be hesitant to prescribe more intensive LLT due to intolerance to statins, potential side effects at higher doses (e.g. rhabdomyolysis or myalgia) and/or lack of awareness of the guidelines' recommendations [12].

The aim of the current study was to evaluate the association of treatment intensity and adherence to LLT (statins and/or ezetimibe) with the risk of major adverse cardiovascular events (MACE) among post-MI patients in Germany.

\section{METHODS}

We conducted a retrospective cohort study using linked data from an anonymised German health claims database, Forschungsdatenbank des InGef-Institut für angewandte Versorgungsforschung Berlin $\mathrm{GmbH}$, which contains data on an estimated 4 million insured individuals from 69 German statutory health 
insurances. Data are standardised to the age and sex distribution of the Federal Statistical Office based on the year 2013 [13]. The database is representative of the overall German population with regards to measures of morbidity, mortality and drug usage [14], but does not include privately insured patients, who represent about $10 \%$ of German population. All data used in the study are explicitly approved by the involved statutory health insurances for health care research usage according to $\$ 287$ or $\$ 75$ of the German social security law.

The study included adult patients ( $\geq 18$ years) who initiated their first LLT between January 1, 2011 and December 31, 2013 , had $\geq 2$ filled prescriptions for a statin or ezetimibe alone or in combination within the first year of treatment, and had documented evidence of MI (Fig. 1). Statin prescription was identified by ATC code (which provides the name of the drug), pack information (which details the number of units in a pack) and the date of billing. In order to determine that the record represented the first initiation of a statin and/or ezetimibe, the year prior to the recorded prescription of a statin and/or ezetimibe was examined to ensure that no statin/ezetimibe was taken during that year. For each patient, the date of the second LLT prescription was defined as the index date. The baseline period for assessing demographics and comorbidities was defined as the period before the index date. The follow-up period then started 1 year after the index date and continued until MACE (hospitalisation for MI, ischaemic stroke or CV death), all-cause death, or the end of follow-up (December 31, 2015), whichever occurred first.

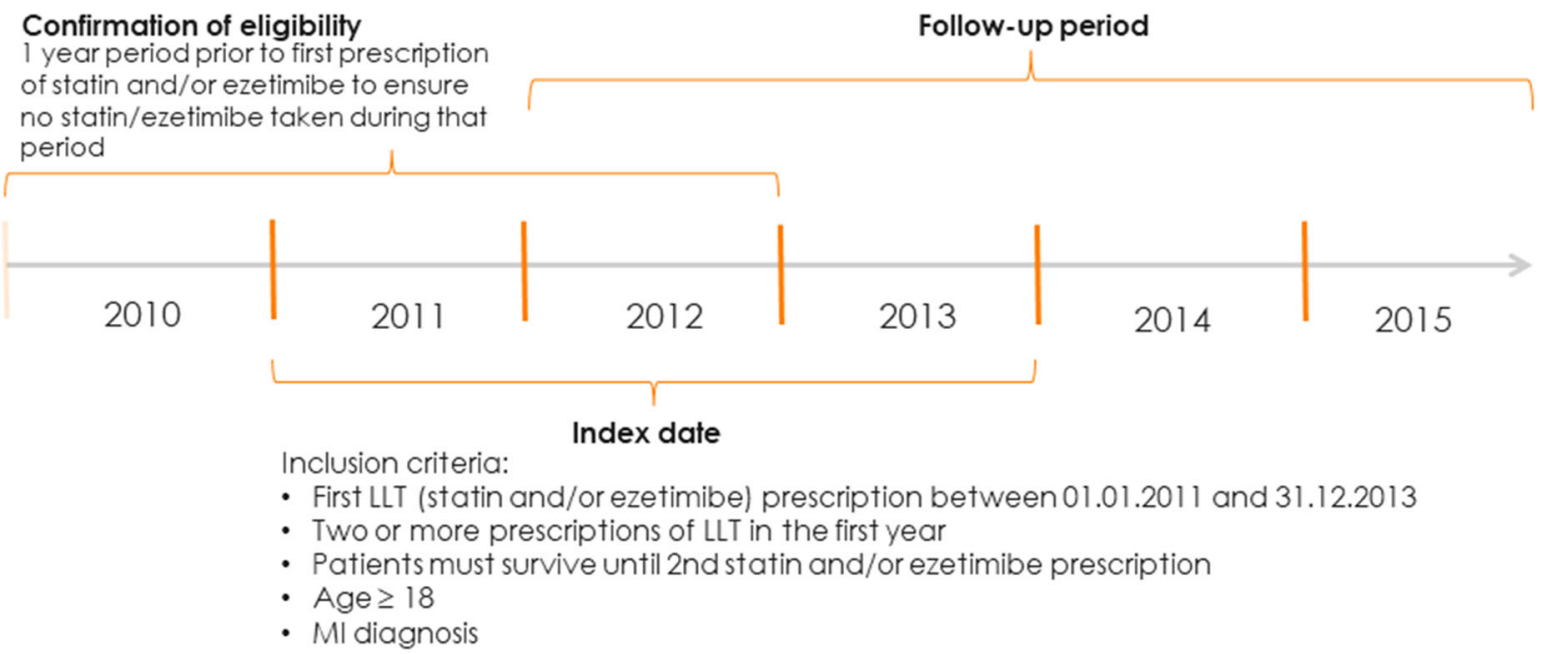

\begin{tabular}{|l|l|l|}
\hline Groups & Adherence, PDC $\geq \mathbf{8 0} \%$ & Non adherence, PDC <80\% \\
\hline $\begin{array}{l}\text { low } \\
\text { intensity }\end{array}$ & $\begin{array}{l}\text { simvastatin } 5 \text { or } 10 \mathrm{mg} ; \text { fluvastatin } 20 \text { or } 40 \mathrm{mg} \text {; lovastatin } 10 \\
\text { or } 20 \mathrm{mg} ; \text { pravastatin } 10,20 \text { or } 30 \mathrm{mg} \text {; pitavastatin } 1 \mathrm{mg} ; \\
\text { ezetimibe monotherapy }\end{array}$ \\
\hline $\begin{array}{l}\text { moderate } \\
\text { intensity }\end{array}$ & $\begin{array}{l}\text { simvastatin } 20,30,40 \text { or } 60 \mathrm{mg} ; \text { atorvastatin } 10,20 \text { or } 30 \mathrm{mg} ; \\
\text { fluvastatin } 80 \mathrm{mg} \text { (including } 40 \mathrm{mg} \text { twice a day); lovastatin } \\
40 \text { or } 80 \mathrm{mg} \text {; pravastatin } 40 \mathrm{mg} \text {; pitavastatin } 2 \text { or } 4 \mathrm{mg} ; \\
\text { rosuvastatin } 5 \text { or } 10 \mathrm{mg}\end{array}$ \\
\hline $\begin{array}{l}\text { high } \\
\text { intensity }\end{array}$ & $\begin{array}{l}\text { simvastatin } 80 \mathrm{mg} ; \text { atorvastatin } 40,60 \text { or } 80 \mathrm{mg} ; \\
\text { rosuvastatin } 20 \text { or } 40 \mathrm{mg} ; \\
\text { all combinations with ezetimibe }\end{array}$ \\
\hline
\end{tabular}

Cox regression for outcome event

Descriptive analysis

Fig. 1 Study design 


\section{Exposures and Outcomes}

LDL-C values were not available in the database, therefore only treatment intensity was used in the analysis. Intensity of statin therapy was classified based on the expected LDL-C reduction as described in the American College of Cardiology and American Heart Association guidelines as well as national guidelines for chronic cardiovascular disease in Germany (Fig. 1) $[13,15,16]$. The classification is also in line with the one used by EUROASPIRE IV survey [4]. The classification of treatment intensity reflected all doses of all statins prescribed in Germany at the time of study. Adherence was measured annually by the proportion of days covered (PDC) using prescription data [17-19]. Patients with a PDC $\geq 80 \%$ were defined as adherent [20].

Treatment intensity and adherence were evaluated as both categorical and continuous variables. As a categorical variable, intensity was based on the most frequently used intensity for the year (i.e. the statistical mode), and was determined annually taking into consideration all doses in a given year; intensity was then categorized as low, moderate or high with regard to the dose and type of LLT used (Fig. 1). Based on its LDL-C lowering effect, as demonstrated in clinical trials [21], ezetimibe monotherapy was considered low intensity, while a combination of a statin with ezetimibe was considered high intensity. Adherence was defined as a categorical variable: non-adherent (PDC $<80 \%$ ) or adherent (PDC $\geq 80 \%$ ). Categorical variables were only used for descriptive purposes. As a continuous variable, intensity was defined based on the expected percent of LDL-C reduction, while adherence was defined as PDC by the therapy. In keeping with a similar earlier study [8], a continuous variable of adherence-adjusted intensity was created by multiplying adherence by treatment intensity variable. This combined measure represented a regimen's intensity after accounting for adherence.

The primary outcome measure was a composite outcome of a MACE, which included hospitalisation for an MI, ischaemic stroke or cardiovascular death. Since cause of death is not recorded in the German database, for the purposes of this study, cardiovascular death was defined as a death that occurred within 30 days after a cardiovascular event.

\section{Statistical Analysis}

Cox proportional hazards regression models were used to evaluate the association of treatment intensity and adherence with the risk of MACE. Three separate models were used to evaluate the continuous variables of treatment intensity, adherence and the combined variable of adherence-adjusted intensity. Intensity and adherence measures were included as timevarying covariates and updated annually. Individuals who received no treatment during the entire year were included in the reference group for that year. Because the adherence and treatment intensity measures, as well as the year of follow-up, were included as time-varying covariates and updated annually, patients moved into and out of the reference group over time as a function of their time-varying covariates.

The following covariates and comorbid conditions were adjusted for and treated as fixed covariates in the models: age (continuous variable), sex (reference: female), history of chronic CVD conditions (any of the following: carotid stenosis, peripheral artery disease, abdominal aortic aneurysm, or other cardiac ischaemia; reference: no), type 2 diabetes (reference: no), chronic kidney disease stage 4-5 (reference: no), atrial fibrillation (reference: no), anti-thrombotic medication use (reference: no), hypertension (reference: no), Charlson Comorbidity Index (reference: 1) and acute cardiovascular hospitalisation in the past year (reference: no). Each model was stratified on covariates that did not meet the proportional hazards assumption. All results were evaluated at a statistical significance level of $\alpha=0.05$ (two-sided). All statistical analyses were conducted using SAS $^{\circledR}$ v.9.4. (SAS Institute, Cary, NC, USA).

\section{Compliance with Ethics Guidelines}

All authors provided substantial contributions to the conception, design of the work, and the 
acquisition, analysis and interpretation of the data for the work. All authors are in agreement to be accountable for all aspects of the work in ensuring the questions related to the accuracy or integrity of any part of the work are appropriately investigated and resolved. All authors give final approval of the version to be published. The Vendor (Elsevier) has permissions to access the data from InGef. The study received access to aggregated anonymised data outputs only, and as such no additional ethical approval was needed.

\section{RESULTS}

A total of 14,944 patients met the study inclusion criteria (Supplementary Figure A) and were followed for a mean of 2.9 [standard deviation $(\mathrm{SD})=1.1]$ years. The patients were on average $66.7(\mathrm{SD}=13.0)$ years old, $68.7 \%$ of patients were men, $68.3 \%$ had a Charlson Comorbidity Index of 2 or more, $89.3 \%$ had hypertension and $93.6 \%$ used antithrombotic medications. At index date, $86.4 \%$ were prescribed moderateintensity statins and only $6.6 \%$ were prescribed high-intensity statins (Table 1 ).

The proportion of patients prescribed highintensity statins increased from 9.3 to $13.2 \%$ over the 4-year follow-up (Table 2). Concurrently, the proportion of patients who discontinued their therapy increased from $9.6 \%$ during the first year of follow-up to $20.7 \%$ during the fourth year. Over $80 \%$ of patients remained on moderate-intensity statins during follow-up. At baseline, $66.8 \%$ of patients were adherent to their LLT (PDC $\geq 0.8$ ). This proportion did not change markedly during the follow-up period, with between 61.7 and $69.5 \%$ of patients on LLT remaining adherent to their regimens (Table 2).

\section{Primary Outcome}

We observed a total of 668 MACE across 44,756 person-years, resulting in a rate of 14.9/1000 person-years. Association of treatment intensity and/or adherence with MACE is shown in Fig. 2. Both intensity and adherence were independently associated with a reduced risk of MACE.
Every $10 \%$ increase in treatment intensity (LDL$\mathrm{C}$ lowering) was associated with a $17 \%$ reduction in risk of MACE [hazard ratio $(\mathrm{HR})=0.83$, 95\% confidence interval (CI) 0.79-0.87]. Similarly, every $10 \%$ increase in adherence was associated with a $5 \%$ reduction in risk of MACE $(\mathrm{HR}=0.95$, 95\% CI 0.94-0.97). When modelling the combined measure of adherence-adjusted intensity, every $10 \%$ increase in adherence-adjusted intensity was associated with a $14 \%$ lower risk of MACE $(\mathrm{HR}=0.86,95 \%$ CI 0.83-0.90) (Fig. 2).

\section{DISCUSSION}

We assessed the association of treatment intensity and adherence to LLT with MACE among post-MI patients in Germany. Among patients with a previous MI, we found that a $10 \%$ increase in intensity, adherence or adherence-adjusted intensity of LLT was associated with $17 \%, 5 \%$ and $14 \%$ lowered risk of MACE, respectively. Treatment intensity was a stronger driver of overall effectiveness of LLT in post-MI patients, as adherence was relatively stable during the follow-up. Our findings are in line with evidence from the Cholesterol Treatment Trialists which found that more intensive treatment led to a $15 \%$ reduction in major vascular events (including coronary death, non-fatal MI and ischaemic stroke) compared to less intensive regimens [9]. Further, in our study, a relatively low proportion of patients were on high-intensity statins, and as, such, the effect of adherence was minimal.

In the present study, no more than $13 \%$ of patients at any time were on high-intensity treatment, which is similar to another German database study that found that between $8 \%$ and $12 \%$ of patients were on a high-intensity statin in a given year [22]. This low use of high-intensity statins does not align with the ESC/EAS guidelines for the management of dyslipidaemia for those at very high risk of cardiovascular events. The ESC/EAS guidelines that were in effect at the time of the study recommended an LDL-C reduction below $1.8 \mathrm{mmol} / \mathrm{L}(70 \mathrm{mg} /$ $\mathrm{dL}$ ) or at least $50 \%$ from baseline LDL-C level [6]. Current ESC/EAS guidelines recommend 
Table 1 Baseline characteristics of the study population $(n=14,944)$

\section{Characteristic}

Age at LLT Initiation, mean (SD) years

$66.7(13.0)$

Sex, $n$ (\%) male

$10,267(68.7 \%)$

Follow-up time, mean $(\mathrm{SD})$ years

$2.9(1.1)$

Charlson Comorbidity Index, $n$ (\%)

1

$4731(31.7 \%)$

$2+$

$10,213(68.3 \%)$

Cardiovascular conditions, $n$ (\%)

Carotid stenosis

$1121(7.5 \%)$

Peripheral artery disease

$1697(11.4 \%)$

Abdominal aortic aneurysm

$247(1.7 \%)$

Other cardiac ischaemia

$14,533(97.3 \%)$

Cardiovascular hospitalisation in the past year, $n$ (\%)

Myocardial infarction

$12,295(82.3 \%)$

Unstable angina

$1211(8.1 \%)$

Ischaemic stroke

$647(4.3 \%)$

Transient ischaemic attack

$176(1.2 \%)$

Revascularisation

$10,775(72.1 \%)$

Type 2 diabetes, $n$ (\%)

$3844(25.7 \%)$

Chronic kidney disease (stage 4 and 5), $n$ (\%)

$497(3.3 \%)$

Atrial fibrillation, $n$ (\%)

$3071(20.6 \%)$

Hypertension, $n$ (\%)

$13,339(89.3 \%)$

Use of antithrombotic medications, $n$ (\%)

$13,981(93.6 \%)$

Initial lipid-lowering therapy, $n$ (\%)

High-intensity statin

$983(6.6 \%)$

Moderate-intensity statin

$12,906(86.4 \%)$

Low-intensity statin

$666(4.5 \%)$

Statin + ezetimibe $^{a}$

$284(1.9 \%)$

Ezetimibe alone $^{\mathrm{b}}$

$105(0.7 \%)$

LLT lipid lowering therapy, $S D$ standard deviation

a Therapy with a statin and ezetimibe was considered high-intensity LLT

b Therapy with ezetimibe alone was considered low intensity LLT 
Table 2 Treatment intensity and adherence, by year of follow-up

\begin{tabular}{|c|c|c|c|c|c|}
\hline & Index year & Year 1 & Year 2 & Year 3 & Year 4 \\
\hline$n$ & 14,944 & 14,338 & 11,716 & 6890 & 2558 \\
\hline On LLT (\%) & $14,944(100 \%)$ & $12,956(90.4 \%)$ & $10,019(85.5 \%)$ & $5695(82.7 \%)$ & $2029(79.3 \%)$ \\
\hline High-intensity ${ }^{\mathrm{a}}(\%)$ & $1391(9.3 \%)$ & $1448(11.2 \%)$ & $1260(12.6 \%)$ & $695(12.2 \%)$ & $267(13.2 \%)$ \\
\hline Moderate-intensity (\%) & $12,787(85.6 \%)$ & $10,830(83.6 \%)$ & $8214(82.0 \%)$ & $4688(82.3 \%)$ & $1659(81.8 \%)$ \\
\hline Low-intensity (\%) & $766(5.1 \%)$ & $678(5.2 \%)$ & $545(5.4 \%)$ & $312(5.5 \%)$ & $103(5.1 \%)$ \\
\hline Not on LLT (\%) & - & $1382(9.6 \%)$ & $1697(14.5 \%)$ & $1195(17.3 \%)$ & $529(20.7 \%)$ \\
\hline Adherent to $\operatorname{LLT}^{\mathrm{b}}(\%)$ & $9989(66.8 \%)$ & $7989(61.7 \%)$ & $6333(63.2 \%)$ & $3637(63.9 \%)$ & $1410(69.5 \%)$ \\
\hline
\end{tabular}

a Proportions of patients on high-, moderate- and low-intensity LLT are calculated out of the number of patients who were on LLT

b Adherence defined as proportion of days covered $\geq 0.8$; proportions are calculated out of those on LLT during each year

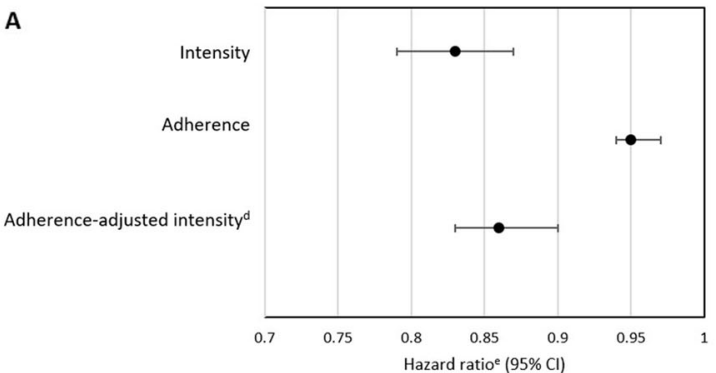

aEstimates are presented per $10 \%$ increase in intensity and/or adherence.

'MACE: composite outcome was defined as hospitalisation due to myocardial infarction, ischaemic stroke or cardiovascular death

Estimated using Cox proportional hazards regression models to evaluate the association of treatment intensity and adherence with the risk of MACE

'Reflects the treatment intensity of a regimen after accounting for a patient's adherence

eHazard ratio after adjusting for age, sex, history of chronic cardiovascular disease conditions, type 2 diabetes, chronic kidney disease stage 4 - 5, atrial fibrillation, use of antithrombotic medications,

hypertension, Charlson Comorbidity Index score and acute cardiovascular hospitalisation in the past year

Treatment intensity range: $0-66 \%$ (as the most intense statin formulation is estimated to lower LDL-C by $66 \%$ ). The combined measure was created by multiplying adherence (range: $0-100 \%$ ) and

treatment intensity (range: $0-66 \%$ ); as a result, the combined measure ranged from 0 to $66 \%$ (i.e., $0.66 \times 1.0=0.66$ )

Fig. 2 Association of treatment intensity and/or adherence with risk of MACE as hazard ratio (a) and by percentage change (b)

even lower treatment goals: $1.4 \mathrm{mmol} / \mathrm{L}$ $(55 \mathrm{mg} / \mathrm{dL})$ and at least $50 \%$ reduction from baseline LDL-C level [1]. As observed in the results of five EUROASPIRE surveys conducted in countries across Europe over the last several years, these treatment goals can rarely be achieved without high-intensity statin therapy, and often require the addition of a non-statin therapy. Moreover, the EUROASPIRE surveys demonstrate that most coronary patients have less than optimal management of LDL-C resulting in a high proportion of patients not achieving the treatment goals recommended by ESC/EAS guidelines [23-25].

Benefits of statins will only be observed if patients adhere to treatment. However, the reasons for suboptimal adherence to statins are not well understood. Hypotheses for lack of adherence include statin intolerance, harmful media reports that doubt the effectiveness of LLT or exaggerate the side effects of statin therapy, and the perception of receiving treatments for asymptomatic conditions [26]. From a patient perspective, adverse events have been 
cited as a common cause of statin discontinuation [27]. A more recent study found that, in patients with $\mathrm{PDC}<50 \%$ versus those with PDC $\geq 80 \%$, the most commonly reported concern of patients regarding statin use was the preference to lower cholesterol with lifestyle changes [28]. Further, the study found that the patients with a lower perceived cardiovascular risk were less likely to be adherent. Though approximately one-third of patients will be adherent to therapy just by being given a prescription by their physician [26], identifying barriers for the remaining patients and providing patient-focussed interventions, such as tailored patient information and education, is necessary to increase adherence.

There are several limitations to our study. As with any retrospective health claims data analysis, data may be subject to coding limitations and data entry errors leading to potential misclassification. However, the misclassification would be non-differential with regards to LLT exposure and, due to the large sample size, the impact of such misclassification on the results is likely to be minimal. Additionally, measures of treatment intensity and adherence were based on filled prescriptions; therefore, it was not possible to ascertain whether the patients actually took the medication as prescribed. This approach, including using PDC as a proxy for adherence, has been used in other studies $[8,17-19]$ and is in line with the guidelines set out by the FDA sentinel program [29]. Not all data elements were available in the database as neither LDL-C levels nor cause of death were included. Therefore, we could not ascertain the effect of LLTs on LDL-C levels in the population. Further, we had to assume that a death that occurred within 30 days of a cardiovascular event was caused by said event. It should also be noted that treatment adherence may be related to factors that are not quantifiable in this analysis, such as lifestyle modifications. Finally, we recognise that the study did not measure the use of all medications that may impact the risk of cardiovascular outcomes.

Our study also has several important strengths. Having relied on a large claims database, our study had a large sample representative of the German population in terms of morbidity, mortality and drug usage [14]. Further, considerable follow-up time allowed for robust estimation of MACE rates. The retrospective observational study design allows for the exploration of benefits of interventions under real-world conditions, thereby increasing the external validity of the results.

\section{CONCLUSION}

The findings of this study highlight the importance of both LLT intensity and adherence in lowering risk of MACE in patients at very high risk of cardiovascular events. The majority of German post-MI patients remain on moderateintensity statin therapy despite the recommendations of the ESC/EAS guidelines for management of dyslipidaemias [7]. Strategies that tailor treatment intensity to patient risk profiles, improve patient adherence to LLTs and ensure that physicians follow the most recent guidelines for reduction of LDL-C, could help optimize lipid management in this population and prevent future cardiovascular events in Germany.

\section{ACKNOWLEDGEMENTS}

We wish to acknowledge Elsevier for providing the data and the conduct of the statistical analysis for this study. Lianne Barnieh, a Certara employee, provided assistance on editing the final manuscript.

Funding. This study was funded by Amgen (Europe) GmbH.

Authorship. All named authors meet the International Committee of Medical Journal Editors (ICMJE) criteria for authorship for this article, take responsibility for the integrity of the work as a whole, and have given their approval for this version to be published.

Disclosures. Eduard Sidelnikov is a full-time employee of Amgen (Europe) $\mathrm{GmbH}$ and holds Amgen stock options. Eugen Dornstauder is a full-time employee of Amgen GmbH, Germany, and holds Amgen stock options. Certara, 
Evidence and Access (Previously-Analytica Laser), employer of Artak Khachatryan, received consulting fees from Amgen (Europe) $\mathrm{GmbH}$ to conduct the study. Elsevier, employer of Bondo Monga, received consulting fees from Certara, Evidence and Access (Previously-Analytica Laser) to conduct the statistical analysis. Ingo Ahrens received consulting fees from Amgen $\mathrm{GmbH}$.

Compliance with Ethics Guidelines. All authors provided substantial contributions to the conception, design of the work, and the acquisition, analysis, and interpretation of the data for the work. All authors are in agreement to be accountable for all aspects of the work in ensuring the questions related to the accuracy or integrity of any part of the work are appropriately investigated and resolved. All authors give final approval of the version to be published. The Vendor (Elsevier) has permissions to access the data from InGef. The study received access to aggregated anonymised data outputs only, and as such no additional ethical approval was needed.

Data Availability. The data underlying this article were provided by Forschungsdatenbank des InGef - Institut für angewandte Versorgungsforschung Berlin $\mathrm{GmbH}$ under licence. Data will be shared on request to the corresponding author with permission of Forschungsdatenbank des InGef - Institut für angewandte Versorgungsforschung Berlin $\mathrm{GmbH}$.

Open Access. This article is licensed under a Creative Commons Attribution-NonCommercial 4.0 International License, which permits any non-commercial use, sharing, adaptation, distribution and reproduction in any medium or format, as long as you give appropriate credit to the original author(s) and the source, provide a link to the Creative Commons licence, and indicate if changes were made. The images or other third party material in this article are included in the article's Creative Commons licence, unless indicated otherwise in a credit line to the material. If material is not included in the article's Creative Commons licence and your intended use is not permitted by statutory regulation or exceeds the permitted use, you will need to obtain permission directly from the copyright holder. To view a copy of this licence, visit http://creativecommons.org/licenses/by$\mathrm{nc} / 4.0 /$.

\section{REFERENCES}

1. Mach F, Baigent C, Catapano AL, Koskinas KC, Casula M, Badimon L, et al. 2019 ESC/EAS Guidelines for the management of dyslipidaemias: lipid modification to reduce cardiovascular risk. Eur Heart J. 2020;41(1):111-88.

2. Townsend N, Wilson L, Bhatnagar P, Wickramasinghe K, Rayner M, Nichols M. Cardiovascular disease in Europe: epidemiological update 2016. Eur Heart J. 2016;37(42):3232-45.

3. Gosswald A, Schienkiewitz A, Nowossadeck E, Busch MA. Prevalence of myocardial infarction and coronary heart disease in adults aged $40-79$ years in Germany: results of the German Health Interview and Examination Survey for Adults (DEGS1). Bundesgesundheitsblatt Gesundheitsforschung Gesundheitsschutz. 2013;56(5-6):650-5.

4. Kotseva K, Wood D, De Bacquer D, De Backer G, Ryden L, Jennings C, et al. EUROASPIRE IV: a European Society of Cardiology survey on the lifestyle, risk factor and therapeutic management of coronary patients from 24 European countries. Eur J Prev Cardiol. 2016;23(6):636-48.

5. World Heart Federation. Cardiovascular risk factors 2020. Available from: https://www.world-heartfederation.org/resources/risk-factors/.

6. Catapano AL, Graham I, De Backer G, Wiklund O, Chapman MJ, Drexel $H$, et al. 2016 ESC/EAS guidelines for the management of dyslipidaemias. Eur Heart J. 2016;37(39):2999-3058.

7. Piepoli MF, Hoes AW, Agewall S, Albus C, Brotons C, Catapano AL, et al. 2016 European guidelines on cardiovascular disease prevention in clinical practice: the Sixth Joint Task Force of the European Society of Cardiology and Other Societies on Cardiovascular Disease Prevention in Clinical Practice (constituted by representatives of 10 societies and by invited experts) developed with the special contribution of the European Association for Cardiovascular Prevention and Rehabilitation (EACPR). Eur Heart J. 2016;37(29):2315-81. 
8. Khunti K, Danese MD, Kutikova L, Catterick D, Sorio-Vilela F, Gleeson M, et al. Association of a combined measure of adherence and treatment intensity with cardiovascular outcomes in patients with atherosclerosis or other cardiovascular risk factors treated with statins and/or Ezetimibe. JAMA Netw Open. 2018;1(8):e185554.

9. Cholesterol Treatment Trialists C, Baigent C, Blackwell L, Emberson J, Holland LE, Reith C, et al. Efficacy and safety of more intensive lowering of LDL cholesterol: a meta-analysis of data from 170,000 participants in 26 randomised trials. Lancet. 2010;376(9753):1670-81.

10. De Vera MA, Bhole V, Burns LC, Lacaille D. Impact of statin adherence on cardiovascular disease and mortality outcomes: a systematic review. Br J Clin Pharmacol. 2014;78(4):684-98.

11. Rasmussen JN, Chong A, Alter DA. Relationship between adherence to evidence-based pharmacotherapy and long-term mortality after acute myocardial infarction. JAMA. 2007;297(2):177-86.

12. Laufs U, Karmann B, Pittrow D. Atorvastatin treatment and LDL cholesterol target attainment in patients at very high cardiovascular risk. Clin Res Cardiol. 2016;105(9):783-90.

13. Aerzteschaft Add. Therapieempfehlungen der Arzneimittelkommission der Deutschen Artzteschaft. 2012.

14. Andersohn F, Walker J. Characteristics and external validity of the German Health Risk Institute (HRI) database. Pharmacoepidemiol Drug Saf. 2016;25(1): 106-9.

15. Stone NJ, Robinson JG, Lichtenstein AH, Bairey Merz CN, Blum CB, Eckel RH, et al. 2013 ACC/AHA guideline on the treatment of blood cholesterol to reduce atherosclerotic cardiovascular risk in adults: a report of the American College of Cardiology/ American Heart Association Task Force on practice guidelines. Circulation. 2014;129(25 Suppl 2):S145 .

16. Nationale VersorgungsLeitlinie. Chronische KHK. 2019.

17. Andrade SE, Kahler KH, Frech F, Chan KA. Methods for evaluation of medication adherence and persistence using automated databases. Pharmacoepidemiol Drug Saf. 2006;15(8):565-74 (discussion 75-7).

18. Anghel LA, Farcas AM, Oprean RN. An overview of the common methods used to measure treatment adherence. Med Pharm Rep. 2019;92(2):117-22.
19. Raebel MA, Schmittdiel J, Karter AJ, Konieczny JL, Steiner JF. Standardizing terminology and definitions of medication adherence and persistence in research employing electronic databases. Med Care. 2013;51(8 Suppl 3):S11-21.

20. Deshpande S, Quek RG, Forbes CA, de Kock S, Kleijnen J, Gandra SR, et al. A systematic review to assess adherence and persistence with statins. Curr Med Res Opin. 2017;33(4):769-78.

21. Cannon CP, Blazing MA, Giugliano RP, McCagg A, White JA, Theroux $\mathrm{P}$, et al. Ezetimibe added to statin therapy after acute coronary syndromes. N Engl J Med. 2015;372(25):2387-97.

22. Fox KM, Tai M-H, Kostev K, Hatz M, Qian Y, Laufs U. Treatment patterns and low-density lipoprotein cholesterol (LDL-C) goal attainment among patients receiving high- or moderate-intensity statins. Clin Res Cardiol. 2018;107(5):380-8.

23. Prugger C, Heidrich J, Wellmann J, Dittrich R, Brand SM, Telgmann R, et al. Trends in cardiovascular risk factors among patients with coronary heart disease: results from the EUROASPIRE I, II, and III surveys in the Munster region. Dtsch Arztebl Int. 2012;109(17):303-10.

24. De Backer G, Jankowski P, Kotseva K, Mirrakhimov E, Reiner Z, Ryden L, et al. Management of dyslipidaemia in patients with coronary heart disease: Results from the ESC-EORP EUROASPIRE V survey in 27 countries. Atherosclerosis. 2019;285:135-46.

25. Reiner Z, De Backer G, Fras Z, Kotseva K, Tokgozoglu L, Wood D, et al. Lipid lowering drug therapy in patients with coronary heart disease from 24 European countries-findings from the EUROASPIRE IV survey. Atherosclerosis. 2016;246: 243-50.

26. Lansberg P, Lee A, Lee ZV, Subramaniam K, Setia S. Nonadherence to statins: individualized intervention strategies outside the pill box. Vasc Health Risk Manag. 2018;14:91-102.

27. Maningat P, Gordon BR, Breslow JL. How do we improve patient compliance and adherence to long-term statin therapy? Curr Atheroscler Rep. 2013;15(1):291.

28. Fung V, Graetz I, Reed M, Jaffe MG. Patient-reported adherence to statin therapy, barriers to adherence, and perceptions of cardiovascular risk. PLoS ONE. 2018;13(2):e0191817.

29. FDA's Sentinel Initiative. Available from: https:// www.fda.gov/safety/fdas-sentinel-initiative. 